\title{
SHORT COMMUNICATION Air-Abrasive Functional Trimming of Thick Film Circuits
}

\author{
L. F. GOULD, R. J. WEST and M. J. WOOD
}

Semiconductor Division, Westinghouse Brake and Signal Co. Limited, Foundry Lane, Chippenham, Wiltshire.

High tolerance chip capacitors are at best expensive and at the worst unobtainable. When asked to custom build a small signal circuit containing close tolerance RC networks it became obvious that functional resistor trimming was unavoidable if suitable quantities were to be produced at the right price.

The product which is shown in Figure 1 is constructed by conventional thick film techniques. It uses a $1^{\prime \prime} \times 2^{\prime \prime}$ alumina substrate and attached components, capacitors, transistors and double diodes. The following points may be of interest:-

1) To enable thick film compatible components to be used, extensive use is made of cross-over techniques. The increased cost due to these cross-overs is small compared to the reduction in assembly costs which these devices allow.

2) The majority of the resistors are trimmed prior to the attachment of the discrete components, using an air abrasive system. The high tolerance, high stability resistors required (top left hand corner of Figure 1) are glazed prior to trimming to increase their stability, to give protection from the encapsulating epoxy, and to warn the trimmer operator that extra care is required.

The circuit produces rectangular pulses of set width at a repetition rate of the order of $15 \mathrm{kHz}$ with a mark space ratio of $5: 1$.

These parameters are determined by the time

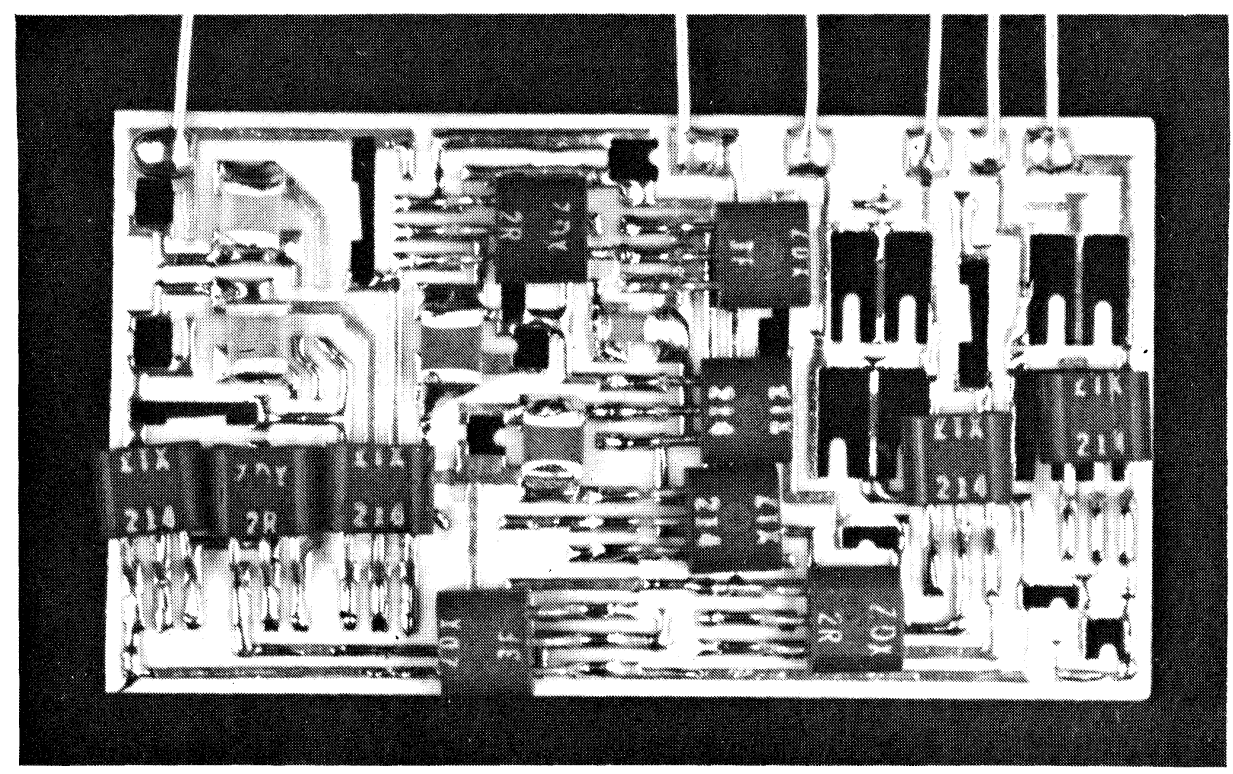

FIGURE 1 Complete thick film hybrid circuit.

$\dagger$ This paper was first presented at the ISHM (U.K.) resistor trimming meeting on 29th November 1973. 


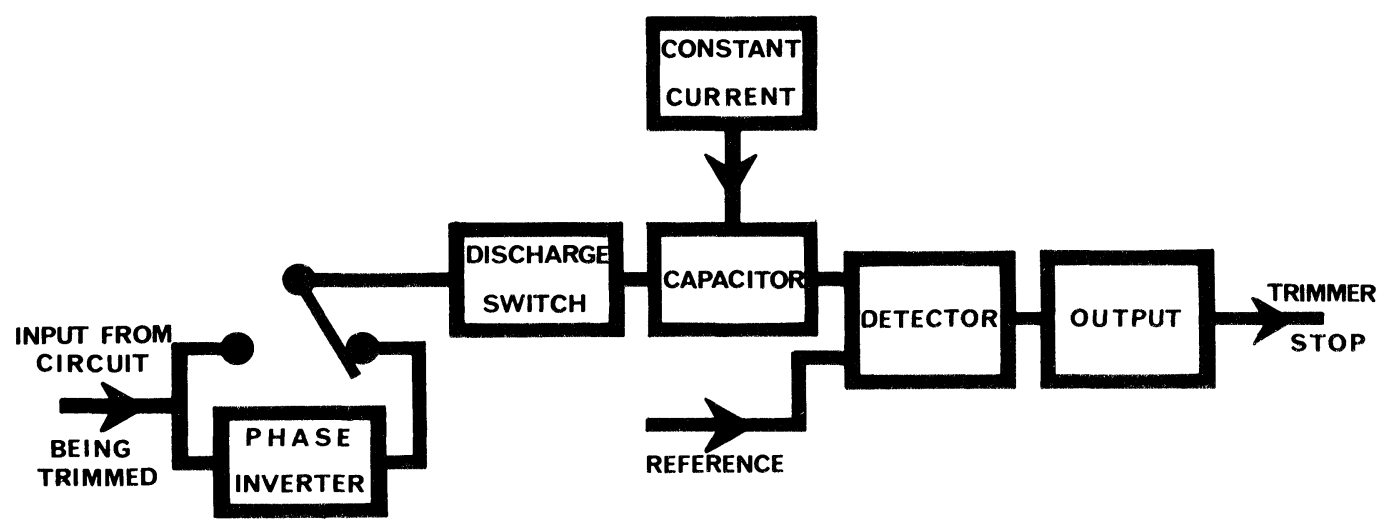

FIGURE 2 Block diagram of pulse measuring circuit used for controlling functional trimming.

constants of an assymetric multivibrator, which contains two $\pm 20 \%$ tolerance NPO (low temperature coefficient of capacitance) capacitors and the two trimmable thick film resistors which are located at an unobstructed end of the substrate. (Top left hand corner Figure 1.)

An MPM RT-5 Air Abrasive trimmer has been modified to allow it to be used for functional trimming. This involves the use of a new jig to hold the circuit and to make electrical contact with its terminals.

The automatic resistance bridge on the RT- 5 is replaced with a pulse measuring circuit which stops the trimming cycle when the correct output is obtained. The block diagram of this circuit is shown in Figure 2.

In order to trim the pulse width the abrasive jet is positioned alongside the appropriate resistor. The output pulses from the circuit under trim, are fed via a phase inverter into the discharge switch. This switch short-circuits the external capacitor shown in Figure 2 , except for the duration of each pulse. The capacitor is supplied from a constant current source and the voltage across it is therefore a linear rising ramp. The height of the ramp depends on the length of time the discharge switch is open, i.e. the pulse width. As the abrasive jet causes the resistor to increase in value, the pulse width and therefore the ramp voltage also increase until the peak ramp voltage is equal to the reference voltage into the detector. The detector then switches and via the output stage stops the cutting action of the trimming machine. The functional trimming set-up therefore trims the resistor to produce a definite pulse width which is pre-set by adjustment of the constant current into the capacitor. In order to trim the space between pulses the abrasive jet is positioned over the other trim-on-test resistor, and the output from the circuit on trim is routed directly (not via the phase inverter) to the discharge switch. The constant current is also switched to a different value to alter the time setting. Because the pulse feeds the discharge switch directly, the switch is now open during the space between pulses and the resistor is therefore trimmed to set the time between each pulse.

The accuracy requirements are pulse width $\pm 10 \%$, space between pulses $\pm 3 \%$. The possible causes of inaccuracy are

1) Drifts in the constant current feeding the ramp capacitor.

2) Variation of supply voltage.

3) Variation of operating time of electronics.

4) Machine delay time (overshoot).

The constant current circuit is temperature compensated by using a dual transistor circuit. Variations due to supply voltage changes are eliminated by making the reference supply proportional to the overall supply voltage, which compensates for changes in constant current due to this cause. The operating time of the detector and output circuit are made very fast compared to the machine cycle time so that errors due to this are minimised. By variation of constant current supply it is possible to cut off early thus avoiding the usual mechanical problem of air abrasive overshoot.

The accuracy requirements of this system have been met easily, allowing full scale production of this module. 

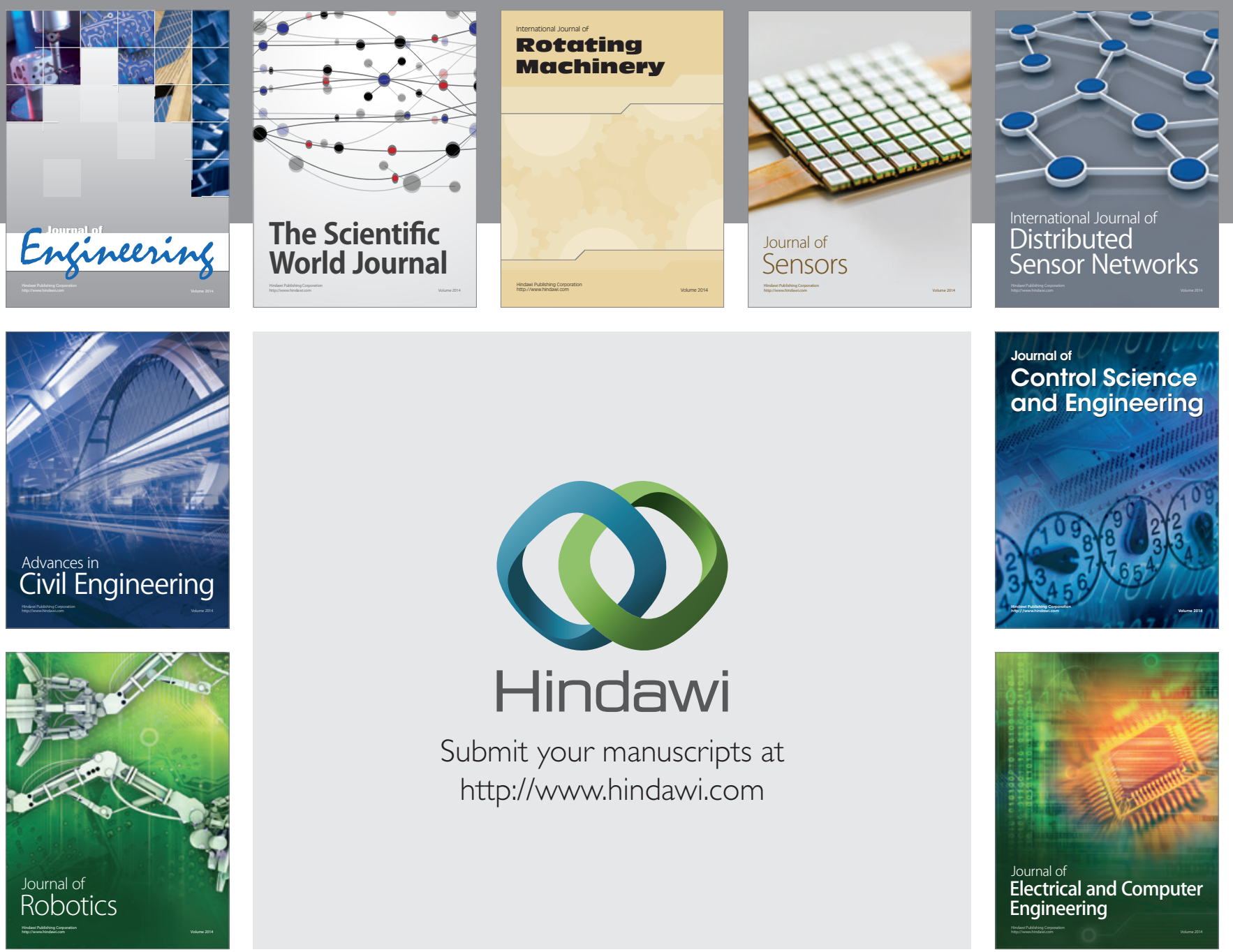

Submit your manuscripts at

http://www.hindawi.com
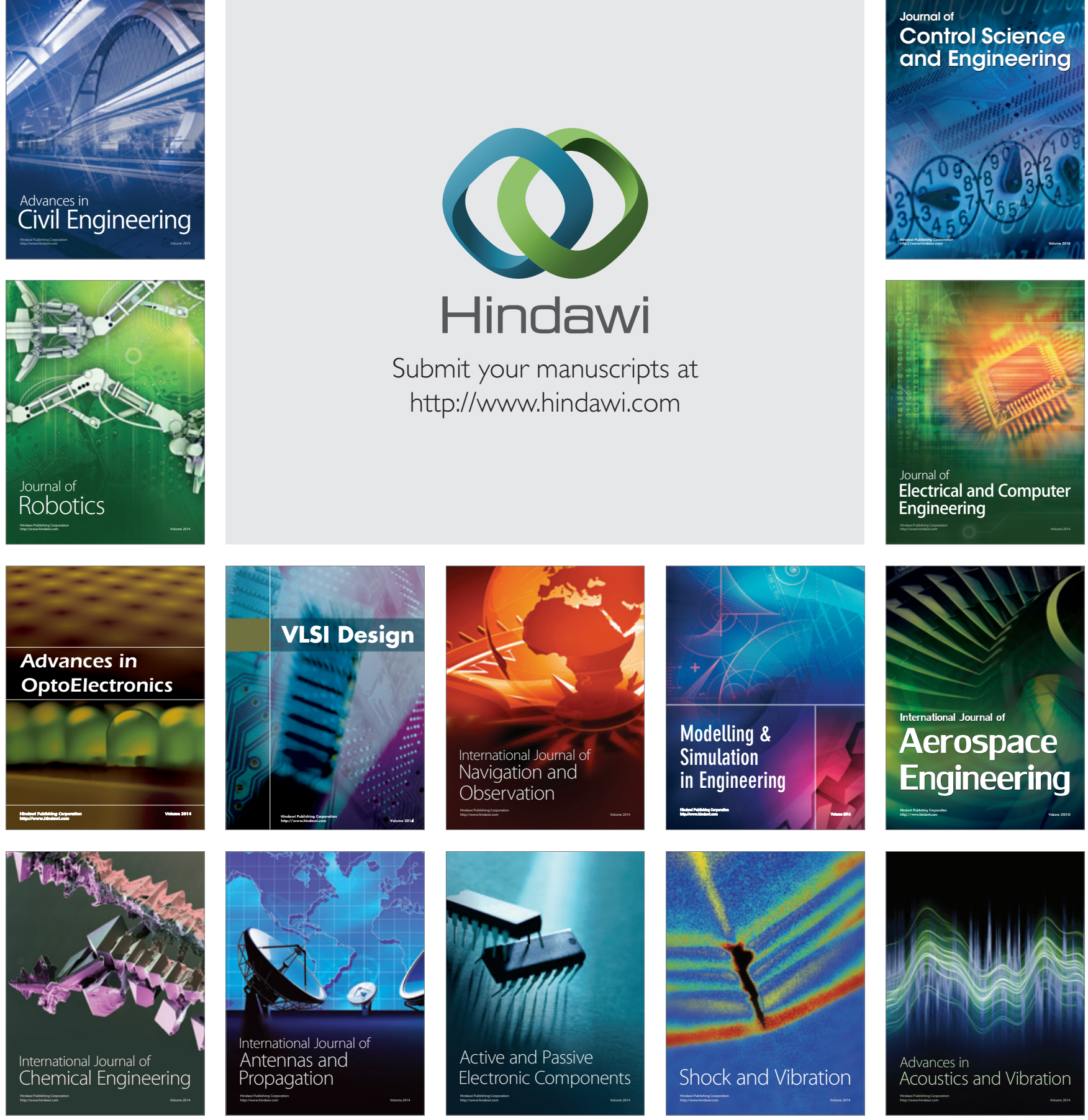